INTERNATIONAL JOURNAL
PHARMACEUTICAL SCIENCES
RESEARCH
REF

Received on 26 December, 2017; received in revised form, 04 March, 2018; accepted, 11 March, 2018; published 01 September, 2018

\title{
STUDIES ON THE EFFECT OF CORE AND SURFACE CROSS-LINKING AGENTS IN PECTIN BEADS ON THE ENTRAPMENT OF INSULIN
}

\author{
V. Prabakaran ${ }^{* 1}$ and N. V. Satheesh Madhav ${ }^{2}$ \\ Jawaharlal Nehru Technological University ${ }^{1}$, Hyderabad - 500072, Telangana, India. \\ Faculty of Pharmacy ${ }^{2}$, DIT University, Dehradun - 248009, Uttarakhand, India.
}

Keywords:

Ionotropic gelling, Internal cross linking agent, Calcium carbonate, Entrapment efficiency

\section{Correspondence to Author:}

\section{Prabakaran}

Research Scholar,

Directorate of Research and

Development, Jawaharlal Nehru

Technological University,

Kukatpally, Hyderabad - 500085,

Telangana, India.

E-mail: prabusuresh@yahoo.co.in

\begin{abstract}
Ionotropic gelation, a characteristic property, has become one of the easy, simple and mild ways of encapsulating variety of drugs, enzymes and proteins, prepared by various polysaccharides. The externally cross linked beads with calcium chloride and zinc chloride were suitable for water soluble and low molecular weight drugs. Insulin, encapsulated in a calcium pectinate gel beads by dispersing the insulin and core cross linking agent in a Solution of pectin, beads are prepared by dropping the dispersion in calcium chloride by inotropic gelation. The purpose of study was to improve the entrapment efficiency of high molecular weight drugs like insulin with internally cross-linking agents like calcium carbonate. Calcium pectinate beads containing insulin was prepared by surface cross linking with $1 \%$ and $5 \% \mathrm{w} / \mathrm{v}$ calcium chloride solution using calcium carbonate as internal gelling agent. Beads obtained using $1 \% \mathrm{w} / \mathrm{v}$ calcium chloride solution showed more drug entrapment than 5\% calcium chloride solution. The core and surface cross linking of pectin beads improves drug loading by about $10-20 \%$ compared to the surface cross linked beads. The beads were characterized by particle size analysis, swelling ratio, SEM, DSC and in-vitro drug dissolution. The current study reveals that the internal cross linking agent marginally helps to improve the entrapment efficiency of insulin in the calcium pectinate beads.
\end{abstract}

INTRODUCTION: The polysaccharides are widely used in oral drug delivery systems because of the simplicity in obtaining desired drug delivery system and drug release profile, to regulate the release of the drug by control of cross-linking, insolubility of crosslinked beads in gastric environment and broad regulatory acceptance. These include sodium alginate, pectin, chitosan, xantan, guar gum, starch, dextran, gellan ${ }^{1-4}$.

\begin{tabular}{|c|c|}
\hline QUICK RESPONSE CODE & DOI: \\
\hline DOI link: http://dx.doi.org/10.13040/IJPSR.0975-8232.9(9).3757-63 \\
\hline
\end{tabular}

Among the various applications, polysaccharides are used for oral controlled release matrices, floating or bioadhesive sustained release beads or tablets, enteric effect, colon targeting of drug and for pulsatile drug release ${ }^{5-9}$. The extent and rate of crosslinking depends on the valency, molecular size, and concentration of cross linking agent, hardening agent and speed and curing time during processing $10-11$. Solubility, molecular size and ionic nature of drug determine the entrapment and drug and drug release. The water soluble and low molecular weight drugs have poor entrapment as compared to insoluble and large molecular weight drugs ${ }^{12}$. To obtain the higher entrapment efficiency in high molecular weight proteins like insulin with the help of internal cross linking agents. 
The drug entrapment and drug release may be governed by extent of surface and core crosslinking of bead, which is function of penetration of cation into the bead, its molecular size and valency (35 grades). The beads structured with closely packed polymer arrangement and egg-box or threedimensional bonding may have different drug holding and releasing abilities. The inclusion of core cross linking may be effective for structuring core of bead and thus enhancing the entrapment efficiency of drug The purpose of present research work was to study effect of core crosslinking and surface crosslinking of pectin on the entrapment efficiency, swelling and drug release of insulin from cross linked pectinate beads. Calcium carbonate and calcium chloride solution was used as core and surface crosslinking agents respectively.

Insulin a high molecular weight drug, widely used in diabetic patients. Insulin is a peptide hormone produced by beta cells in the pancreas. It regulates the metabolism of carbohydrates and fats by promoting the absorption of glucose from the blood to skeletal muscles and fat tissue and by causing fat to be stored rather than used for energy. The beads were evaluated for micromeritic properties, entrapment efficiency surface topography and differential scanning calorimetry, swelling study and dissolution study.

\section{MATERIALS AND METHODS:}

Materials: Pectin (LM-104 AS) was the generous gift from CPKelco Pvt. Ltd. (Mumbai, India) Insulin was purchased from the marketed product. Calcium chloride purchased from Sisco Research Lab. Pvt. Ltd. (Mumbai, India). All other chemicals were of analytical reagent grade.

\section{Methods:}

Preparation of Insulin Loaded Calcium Pectinate Beads: The beads prepared by the ionotropic gelation method ${ }^{13}$. The $10 \mathrm{ml}$ of pectin solutions of different concentrations by dissolving LM pectin in distilled water with gentle agitation Table 1 . Insulin 10 IU was dispersed pectin solution with constant stirring for 2 min for uniform distribution. The resultant dispersions were pumped through spray gun into $200 \mathrm{ml}$ of stirred calcium chloride solution $(1 \%$ and $5 \% \mathrm{w} / \mathrm{v})$ at room temperature. The flow rate was controlled by the peristaltic pump (approximately $4 \mathrm{ml} / \mathrm{min}$ ).
The beads formed were allowed to remain in the stirred $\mathrm{CaCl}_{2}$ for $10 \mathrm{~min}$ for curing time, filtered and washed with distilled water. Then the beads were dried at room temp for $24 \mathrm{~h}$. Such beads termed as 'Surface cross linked beads'.

\section{Preparation of Pectin Beads with Core Cross} Linking Agent: Beads containing core cross linking agent were prepared by dispersing the insulin $10 \mathrm{IU}$ and $10 \mathrm{mg}$ of $\mathrm{CaCO}_{3}$ in different concentration and stirred $2 \mathrm{~min}$ for uniform distribution. Then the beads were prepared by the above method

\section{Characterization of the Beads:}

Particle Size: The mean diameter was determined by using a stereomicroscope (Carl Zeiss, Germany) attached with a digital camera (Watec, Wat-202, Japan). The captured images were analyzed by using Biovis Image Plus software (Expert Tech Vision, India). About 20 particles of each batch were analyzed and average diameter and different surface factors such as circulatory factor, elongation, roundness and perimeter ratio was determined.

Encapsulation Efficiency: The weights of dried beads were considered as practical yield of the process. $100 \mathrm{mg}$ of the insulin-loaded beads were dissolved in phosphate buffer $\mathrm{pH} 7.4$ by shaking on rotary shaker (Steelmet Industries, Pune, India) at $200 \mathrm{rpm}$ for $1 \mathrm{~h}$. The solution was filtered using 0.45 -micron pore size filter and after sufficient dilution with phosphate buffer $(\mathrm{pH} 7.4)$ analyzed spectrophometrically at $220 \mathrm{~nm}$ (Jasco V500, Japan). The determinations were made in triplicate. The ratio of the actual Insulin content in the drugloaded beads to the theoretical Insulin content was termed the encapsulation efficiency was calculated by equation 1 :

Encapsulation Efficiency =

$$
\frac{\text { Amount of encapsulated drug }}{\text { Amount of added drug }} \times 100
$$

Encapsulation efficiency difference $\left(\mathrm{EE}_{\mathrm{diff}}\right)$ is the difference in encapsulation efficiency of beads prepared using $1 \%$ and $5 \% \mathrm{w} / \mathrm{v}$ of calcium chloride solution.

Surface Topography: The beads were mounted on the standard specimen mounting stubs and were coated with a thin gold-palladium layer $(20 \mathrm{~nm})$ in 
sputter coater unit (VG Microtech, UK). Microphotographs of the beads were observed at 50X and 200X magnification using Cambridge Stereoscan 120 scanning electron microscope (Cambridge UK) operated with an acceleration voltage of $10 \mathrm{kV}$.

Differential Scanning Calorimetry: Thermograms of insulin, calcium pectinate beads without drug, and drug-loaded beads were obtained using a Mettler- Toledo DSC $821^{\mathrm{e}} \quad$ (Switzerland) instrument equipped with an intracooler. Indium standard was used to calibrate the DSC temperature and enthalpy scale. The powder samples were hermetically sealed in perforated aluminum pans and heated at constant rate of $10^{\circ} \mathrm{C} / \mathrm{min}$ over a temperature range of $25-300{ }^{\circ} \mathrm{C}$. The system was purged with nitrogen gas at the rate of $100 \mathrm{ml} / \mathrm{min}$ to maintain inert atmosphere.

Swelling Study: It was carried out by placing beads of each batch in wire basket of USP dissolution apparatus II in baskets containing 500 $\mathrm{ml}$ of $0.1 \mathrm{~N} \mathrm{HCl}(\mathrm{pH} 1.2)$ maintained at $37^{\circ} \mathrm{C}$. The beads were periodically removed at predetermined intervals during study period $2 \mathrm{~h}$ and weighed before and during the swelling. Then the swelling ratio was calculated as per following formula:

Swelling ratio $=\frac{\text { Weight of wet beads }}{\text { Weight of dry beads }}$

Dissolution Studies: The dissolution of Insulin loaded calcium pectinate beads was studied using USP 26 Type II dissolution test apparatus (Electrolab TDT-06P, India) containing $900 \mathrm{ml}$ of $0.1 \mathrm{~N} \mathrm{HCl}\left(\mathrm{pH} \mathrm{1.2)}\right.$ maintained at $37 \pm 0.5^{\circ} \mathrm{C}$ and stirred at $100 \mathrm{rpm}$. Samples were collected periodically and replaced with a fresh dissolution medium. After filtration through whatman filter paper, concentration of insulin was determined by spectrophotometrically (Jasco-V500, Japan) at 220 $\mathrm{nm}$. Analysis of data was done using 'PCP Disso v2.08' software, (Poona College of Pharmacy, Pune, India). All the readings were done in triplicate.

RESULTS AND DISCUSSION: Beads of pectin containing insulin were prepared by inotropic gelation, which form intermolecular crosslinking between divalent calcium ions and negatively charged carboxyl group of pectin molecules.
Divalent metals establish direct polyanion-cationpolyanion interaction between pairs of carboxylic groups on neighbouring helices producing egg-box model ${ }^{14}$. Primary batches of drug-loaded pectin beads containing $3 \% \mathrm{w} / \mathrm{v}$ pectin produced satisfactory beads and it was selected as minimum concentration. The $8 \% \mathrm{w} / \mathrm{v}$ pectin concentration was taken as upper concentration above which the solution was viscous to drop. Aqueous solution of 1 and $5 \% \mathrm{w} / \mathrm{v}$ calcium chloride was used as surface crosslinking agent to ensure relative calcium reactivity.

Significant differences were observed in the encapsulation efficiency of thus formed batches. Entrapment efficiency was in the range of 38 to $88 \%$ as shown in Table 1. The encapsulation efficiency of surface crosslinked beads increased with the increase in the pectin concentration but decreased with increasing the calcium chloride concentration from $1 \%$ to $5 \% \mathrm{w} / \mathrm{v}$. The calculated entrapment efficiency difference ( $\left.\mathrm{EE}_{\text {diff }}\right)$ was 14 to $21 \%$ except batch AG-3 containing lowest polymer content. The increase in entrapment efficiency with increase in pectin concentration at constant drug amount may be attributed to the availability of excess polymer to encapsulate the drug. The reduction in entrapment efficiency of these batches in $5 \%$ calcium chloride solution may be attributed to weakening of surface gel strength due to excess of $\left(\mathrm{Ca}^{2+}\right)$. The lower value of $\mathrm{EE}_{\text {diff }}$ for $3 \%$ pectin containing batches can be correlated to lower amount of polymer and higher $\left(\mathrm{Ca}^{2+}\right)$ : polymer ratio.

The entrapment efficiency was further increased in batches containing calcium carbonate as core/ internal gelling agent. In the internally gelled batches containing $3 \% \mathrm{w} / \mathrm{v}$ pectin there was almost $24 \%$ and $20 \%$ increase in entrapment efficiency using $1 \%$ and $5 \% \mathrm{w} / \mathrm{v}$ calcium chloride solution respectively. The other internally gelled batches containing higher pectin concentrations and surface crosslinked using $1 \% \mathrm{w} / \mathrm{v}$ calcium chloride solution showed the improvement in drug entrapment in range of 5 to $16 \%$ and it was inverse to pectin concentration. As compared with only surface crosslinked batches the drug loss in $5 \% \mathrm{w} / \mathrm{v}$ calcium chloride solution was greatly reduced by inclusion of core crosslinking agent. 
This whole phenomenon reflects the physical and chemical interactions that are associated during formation of pectin gel bead. The weak and flexible gel turns strong and rigid as the availability of cations increases with maximum gel strength on utilization of all possible crosslinking sites. When excess of divalent cations are present, they compete for interaction with anionic sites imposing repulsive forces resulting in weakening of gels ${ }^{13}$.

In surface crosslinked beads, the poor diffusion of large $\mathrm{Ca}^{2+}$ in gel structure causes more surface crosslinking as compared towards core giving rise to increased dissolution / leaching of small and low molecular weight drug through the crosslinked network. It is supported by the high values of

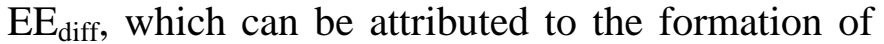
soft gel due to presence of excessive calcium. The increase in drug entrapment and reduced values of $\mathrm{EE}_{\text {diff }}$ in internally gelled batches showed the dominance of core crosslinking over the surface one. The inter molecular cohesion occurred during core crosslinking might have counteracted the weakening of gel making both the core and surface closely compact. This can be supported by reduced drug loss in 5\% w/v crosslinking solution. While as in $1 \%$ surface crosslinking solution the ratio between the calcium ions and the increasing polymer amount played a crucial role, as the polymer concentration increased thereby minimizing the effect of internal crosslinking agent.

\begin{tabular}{|c|c|c|c|c|c|}
\hline AG- 3 & 3 & & $38.44 \pm 1.2$ & $39.52 \pm 1.14$ & 1.08 \\
\hline AG- 5 & 5 & & $69.38 \pm 2.6$ & $53.99 \pm 1.02$ & 15.39 \\
\hline AG- 5 (I) & 5 & 10 & $87.07 \pm 1.54$ & $79.26 \pm 3.14$ & 7.81 \\
\hline AG- 6 & 6 & & $74.68 \pm 1.86$ & $55.18 \pm 3.25$ & 19.5 \\
\hline AG-7 & 7 & & $78.38 \pm 1.60$ & $63.88 \pm 2.55$ & 14.5 \\
\hline AG-7 (I) & 7 & 10 & $91.76 \pm 1.52$ & $88.18 \pm 2.31$ & 3.58 \\
\hline AG-8 & 8 & & $87.24 \pm 1.53$ & $66.03 \pm 1.66$ & 21.21 \\
\hline AG-8 (I) & 8 & 10 & $94.90 \pm 1.61$ & $93.43 \pm 0.09$ & 1.47 \\
\hline
\end{tabular}

EE: \% Encapsulation efficiency, $\mathrm{EE}_{\mathrm{diff}} \%$ Encapsulation efficiency difference

SEM photographs explain surface morphology of dried Insulin loaded Ca-pectinate beads Fig. 1. Batch AG 3-1, surface crosslinked drug loaded beads obtained using low polymer and low calcium chloride concentration yielded soft beads. The bead surface showed prominent thin ridges by "pullaway' effect producing rough surface Fig. 1: A and A1. The beads containing same pectin concentration but prepared using $5 \%$ crosslinking solution was deformed with cavities on the surface (AG-3 B and B1). This can be correlated to loss of gel strength in presence of high $\left(\mathrm{Ca}^{2+}\right)$.

The inclusion of internal gelling agent produced spherical beads with smooth surface $\mathrm{C}$ and $\mathrm{C} 1$ of batch (AG 3-1 (I), C and C1). The beads containing higher pectin were spherical and covered with thick gel layer. Fine drug crystals were appeared on surface of beads prepared using 5\% calcium chloride solution (AG 3-5 (I), D and D1). The surface of beads contained core crosslinking agent showed fewer drug crystals than beads obtained by only surface crosslinking that reconfirms the reduced leaching of drug solution (B1 and D1). The mean particle size of the beads were between 2.073 $\pm 0.057 \mathrm{~mm}$ and $4.376 \pm 0.075 \mathrm{~mm}$. The beads obtained were also evaluated for circulatory factor and roundness. The beads were spherical with circulatory factor in range of 1.2 to 2.0 and roundness in range of 0.35 to 0.8 . In surface crosslinked batches the mean particle size of the beads containing constant drug amount increased with increase in polymer concentration. Using $5 \%$ w/v calcium chloride concentration it showed erratic pattern on increase in size of bead.

DSC thermograms of insulin, unloaded beads and drug loaded calcium pectinate beads of batch NV 5 are shown Fig. 2. Insulin showed melting endotherms at $160.79{ }^{\circ} \mathrm{C}\left(160.44-167.89{ }^{\circ} \mathrm{C}\right)$. Empty pectin beads had two melting endotherms and drug loaded beads depicted three endotherms. The broad endotherm at $90-115^{\circ} \mathrm{C}$ may be due to water loss, peak intensity of that decreases in 
internally crosslinked beads. The shift of melting endotherm of drug to higher temperature observed in crosslinked beads may be attributed to slower heat transfer with increase in hardness of the bead.
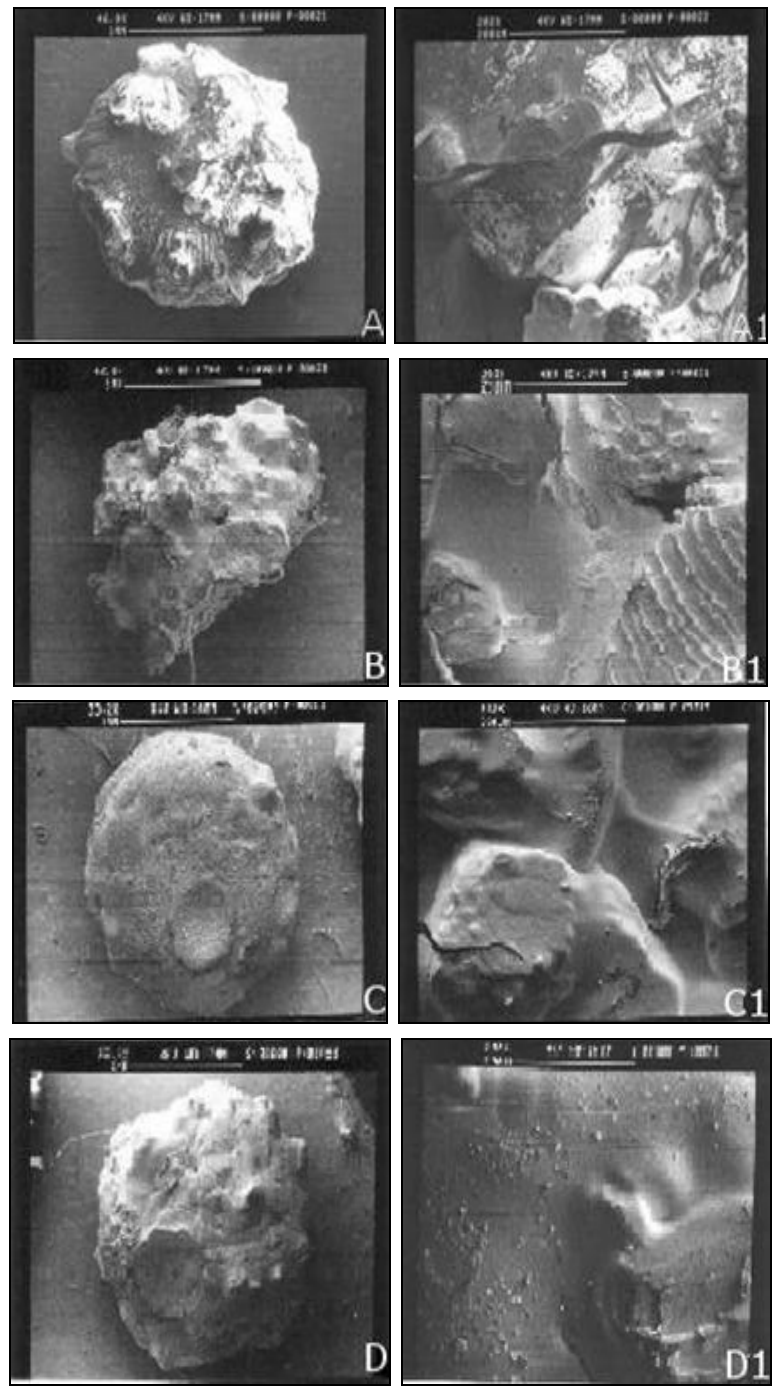

FIG. 1: SCANNING ELECTRON MICROSCOPY OF INSULIN LOADED CALCIUM PECTINATE BEADS

A and A1 of batch AG 3-1, B and B1 of batch AG 3- 5, C and C1 of batch AG 3-1 (I), D and D1 of batch AG 5(I).

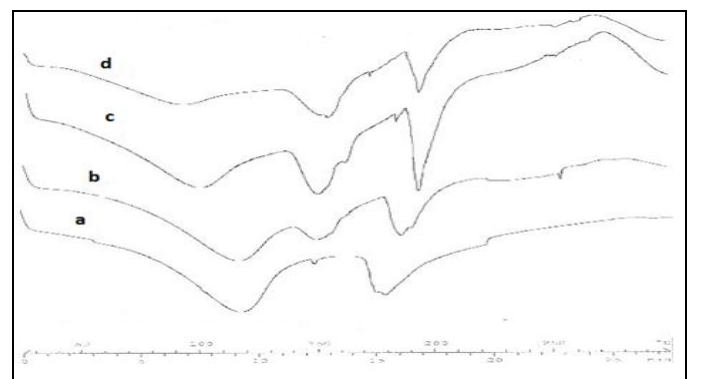

FIG: 2: DSC THERMO GRAMS OF a) INSULIN, b) UNLOADED BEADS c) CALCIUM PECTINATE BEADS AG-5, D) CALCIUM PECTINATE BEADS AG-5I

For swelling studies the dried beads were immersed in $0.1 \mathrm{~N} \mathrm{HCl}$ for two $h$. The maximum swelling was observed with in 20 min. The beads containing $5 \%$ pectin concentration showed high swelling ratio and slower erosion, which indicated optimum amount of pectin and $\left[\mathrm{Ca}^{2+}\right]$ for crosslinking. Beads of AG3-5 showed rapid swelling with shortest plateau Fig. 3.

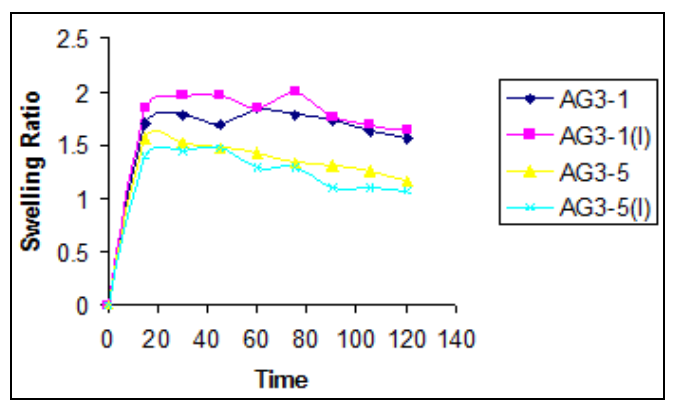

FIG. 3: SWELliNG RATIO OF CA- PECTINATE BEADS: BATCH AG3

It may be attributed to faster penetration of fluid and erosion due to weak gel strength. The other batches containing higher pectin concentration showed somewhat similar swelling profile with swelling ratio around 2 and no erosion within $2 \mathrm{~h}$. The beads obtained by internal crosslinking had lower swelling capacity Fig. 4.

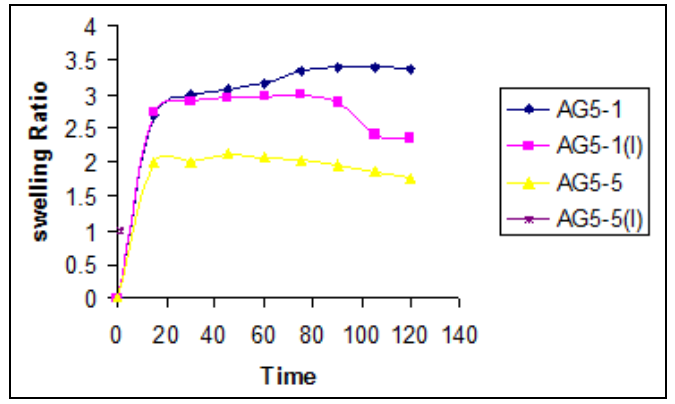

FIG. 4: SWELLING RATIO OF CA-PECTINATE BEADS: BATCH AG5

The dissolution studies of the beads were carried out in $0.1 \mathrm{~N} \mathrm{HCl}(\mathrm{pH} 1.2)$. All the beads showed almost $80 \%$ drug release within 30 to $60 \mathrm{~min}$.

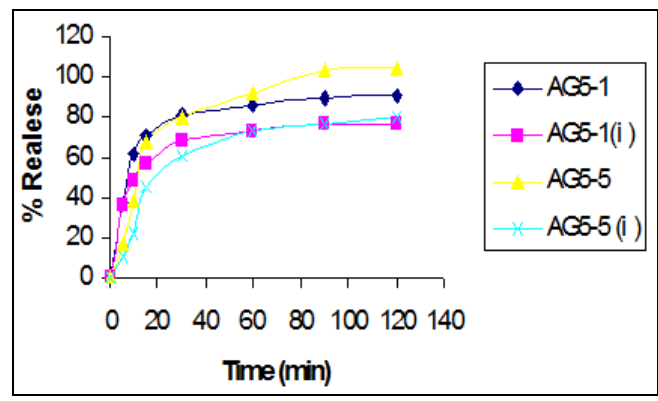

FIG. 5: EFFECT OF INTERNAL AND EXTERNAL CROSS LINKING ON DRUG RELEASE PROFILE 
The drug release was dependent on drug solubility in dissolution medium, because the fluid migrates into the beads to dissolve drugs and create pores and channels. Fluid ingress promotes the extent and the rate of beads swelling creates large surface area, which in turn enhances the release of incorporated drugs.
A typical drug release profile is shown in Fig. 5 and the collective release at different times is given in Table 2. The initial drug release from beads prepared using $5 \% \mathrm{w} / \mathrm{v}$ calcium chloride solution was slow than those prepared using $1 \% \mathrm{w} / \mathrm{v}$ calcium chloride solution. The internal gelling also reduced percent drug release from the beads.

TABLE 2: PERCENT RELEASE PROFILE OF CALCIUM PECTINATE BEADS AT VARIOUS TIME INTERVALS

\begin{tabular}{cccccccc}
\hline \multirow{2}{*}{$\begin{array}{c}\text { Pectin } \\
\text { concentration } \\
\text { Pectin }\end{array}$} & $\begin{array}{c}\text { Crosslinking } \\
\text { agents }\end{array}$ & \multicolumn{6}{c}{ \% Release } \\
\cline { 3 - 8 } concentration & $\begin{array}{c}\text { Crosslinking } \\
\text { agents }\end{array}$ & $\begin{array}{c}\mathbf{1 0} \\
\mathbf{m i n}\end{array}$ & $\begin{array}{c}\mathbf{3 0} \mathbf{C a C l}_{\mathbf{2}} \\
\mathbf{m i n}\end{array}$ & $\begin{array}{c}\mathbf{6 0} \\
\text { min }\end{array}$ & $\begin{array}{c}\mathbf{1 0} \\
\text { min }\end{array}$ & $\begin{array}{c}\mathbf{3 0} \\
\text { min }\end{array}$ & $\begin{array}{c}\mathbf{6 0} \\
\text { min }\end{array}$ \\
\hline $3 \%$ & Cal. chloride & $49.88 \pm 2.4$ & $77.59 \pm .7$ & $91.96 \pm 3.2$ & $54.53 \pm 5.6$ & $81.73 \pm 4.3$ & $92.96 \pm 2.5$ \\
& Cal. carbonate (I) & $44.52 \pm 2.1$ & $68.07 \pm 2.6$ & $79.4 \pm 3.9$ & $43.9 \pm 5.9$ & $67.0 \pm 6.2$ & $85.61 \pm 5.6$ \\
$5 \%$ & Cal. chloride & $61.44 \pm 4.8$ & $82.79 \pm 6.8$ & $86.16 \pm 7.2$ & $38.48 \pm 5.3$ & $80.12 \pm 8.2$ & $92.4 \pm 2.9$ \\
& Cal. carbonate (I) & $49.44 \pm 3.6$ & $68.65 \pm 3.8$ & $73.93 \pm 2.9$ & $21.94 \pm 6.3$ & $60.52 \pm 5.8$ & $83.85 \pm 5.9$ \\
$6 \%$ & Cal. chloride & $55.20 \pm 2.9$ & $73.64 \pm 5.4$ & $89.27 \pm 5.9$ & $45.11 \pm 5.6$ & $89.08 \pm 6.3$ & $96.63 \pm 2.9$ \\
& Cal. carbonate (I) & $31.8 \pm 2.6$ & $54.08 \pm 2.8$ & $85.38 \pm 2.8$ & $63.28 \pm 3.8$ & $72.52 \pm 5.7$ & $87.18 \pm 5.9$ \\
$7 \%$ & Cal. chloride & $53.01 \pm 5.3$ & $78.26 \pm 4.6$ & $81.28 \pm 5.6$ & $35.97 \pm 4.6$ & $87.06 \pm 4.3$ & $93.26 \pm 4.3$ \\
& Cal. carbonate (I) & $38.19 \pm 2.9$ & $56.57 \pm 3.6$ & $88.14 \pm 5.6$ & $43.77 \pm 3.9$ & $63.74 \pm 3.6$ & $78.48 \pm 2.9$ \\
$8 \%$ & Cal. chloride & $65.33 \pm 5.6$ & $82.86 \pm 5.3$ & $85.67 \pm 7.2$ & $42.37 \pm 4.6$ & $83.59 \pm 5.8$ & $88.01 \pm 7.6$ \\
& Cal. carbonate (I) & $33.28 \pm 2.6$ & $60.71 \pm 6.2$ & $78.99 \pm 6.7$ & $36.98 \pm 5.3$ & $60.15 \pm 4.6$ & $77.06 \pm 8.3$ \\
\hline
\end{tabular}

CONCLUSION: In conclusion, the pharmaceutical properties of the ionically crosslinked polysaccharide beads are not only determined by concentration and valency of the cation but the uniform internal and external crosslinking. The core and surface crosslinking of beads can produce closely packed beads with reduced leaching of drug during processing and drying. Thus this study emphasized for the use of internal crosslinking agents to obtain crosslinked calcium-pectinate beads to be promising for the improved drug entrapment, size and shape.

ACKNOWLEDGEMENT: This study was a part of my Ph.D research carried out under the guidance of Dr. N. V. Satheesh Madahv, Director, DITDehradun. I am ever indebted to him for the timely guidance, excellent ideas, pragmatic criticism and the moral support extended to me.

CONFLICT OF INTEREST: The authors have declared that there is no conflict of interest.

\section{REFERENCES:}

1. Nachiket P, Darshan L, Steven G, Elisha I, Youssef S and Jerry N: Development and evaluation of a calcium alginate based oral ceftriaxone sodium formulation. Progress in Biomaterials 2016; 5: 117-133.

2. Bhatia S: Systems for drug delivery. Springer International Publishing Switzerland IX Chapter-2, 2016; 29: 29-54.
3. Zafar A, Bhattacharyya A, Bajpai.M, Yasir M and Asif M: Formulation and in-vitro characterization of floating gel beads of metformin hydrochloride. International Journal of Pharmaceutical Sciences and Nanotechnology 2014; 1: 2356-2362.

4. Siddhartha $\mathrm{M}$ and Biswanath $\mathrm{S}$ : Development and evaluation of $\mathrm{Ca}^{+2}$ ion cross-linked carboxymethyl xanthan gum tablet prepared by wet granulation technique. AAPS Pharm Sci Tech 2014; 15: 920-927.

5. Li L, Wang L, Li J, Jiang S, Wang Y, Zhang X, Ding J, Yu $\mathrm{T}$ and Mao $\mathrm{S}$; Insights into the mechanisms of chitosananionic polymers-based matrix tablets for extended drug release. International Journal of Pharmaceutics 2014; 476: 253-65.

6. Mahboubeh R, Shaik N, Hamed K, Soheil Z and Mohamed I: Hydrogel polysaccharides of tamarind and xanthan to formulate hydro dynamically balanced matrix tablets of famotidine. Molecules 2014; 19: 13909-13931.

7. Asif H, Sabiha S, Sujan B, Masud K, Selim R and Mohammad S: Formulation and in-vitro characterization of hydrochlorothiazide gastroretentive floating drug delivery system. Dhaka University Journal of Pharmaceutical Sciences 2015; 14:163-170.

8. Vivek R and Rachna K; Polysaccharides in colon-specific drug delivery. International Journal of Pharmaceutics 2001; 224:19-38.

9. Rakesh P, Shiv B, Vipin K and Kanchan K: Role of natural polymers in the development of floating drug delivery systems. Journal of Pharmacy Research 2010; 3: 1312-1318.

10. Sapana P, Paraag S, Shrivastav and Pankaj S: Ionotropic Gelation: A Promising crosslinking technique for hydrogels. Research and Reviews: Journal of Pharmaceutics and Nano-technology 2014; 2: 1-6.

11. Avinash Y, Ajay K and Ajay G: Role of excipients and polymeric advancements in preparation of floating drug delivery systems. International Journal of Pharmaceutical Investigations 2015; 5: 1-12. 
12. Rajesh $\mathrm{S}$ and James W: Nanoparticle-based targeted drug delivery. Experimental and Molecular Pathology 2009; 86: 215-223.

13. Takka $\mathrm{S}$ and Acartürk F: Calcium alginate microparticles for oral administration: I: Effect of sodium alginate type on drug release and drug entrapment efficiency. Journal of Microencapsulation 1999; 16: 275-90.

14. Prabakaran V and Satheesh M: The effect of different concentration of pectin solution in calcium pectinate beads on the entrapment of insulin. World journal of pharmaceutical research 2017; 6: 539-547.

How to cite this article:

Prabakaran V and Madhav NVS: Studies on the effect of core and surface cross-linking agents in pectin beads on the entrapment of insulin. Int J Pharm Sci \& Res 2018; 9(9): 3757-63. doi: 10.13040/IJPSR.0975-8232.9(9).3757-63.

All @ 2013 are reserved by International Journal of Pharmaceutical Sciences and Research. This Journal licensed under a Creative Commons Attribution-NonCommercial-ShareAlike 3.0 Unported License.

This article can be downloaded to ANDROID OS based mobile. Scan QR Code using Code/Bar Scanner from your mobile. (Scanners are available on Google Playstore) 